\title{
EVALUATION OF MEIBOMIAN GLANDS LOSS AND TEAR FILM BREAKUP TIME IN PATIENTS WITH VARYING PERIOD OF SOFT CONTACT LENSES WEARING
}

\author{
M. Dimitrova ${ }^{1 *}$, St. Kostova ${ }^{2}$, A. Vassilev ${ }^{2}$, N. Dakov ${ }^{2}$, B. Irinkova ${ }^{1}$, A. Oscar ${ }^{1}$ \\ ${ }^{1}$ Eye Clinic "Zora", Sofia, Bulgaria \\ ${ }^{2}$ Department of Ophthalmology, Medical University-Sofia, Alexandrovska Hospital, Sofia, Bulgaria
}

\begin{abstract}
Purpose: To determine whether the wearing time of contact lenses affects the condition of the meibomian glands and the tear film breakup time.

Material and methods: This is a cross-sectional study involving 150 eyes ( 75 patients) with an age between 18 to 65 years. Five groups were allocated, with distribution criteria being the soft contact lenses wearing time: up to 5 years, from 6 to 10 years, from 11 to 20 years and more than 20 years. All subjects used soft contact lenses daily, at least 5 hours a day and 4 days a week. All patients underwent a thorough ophthalmic examination and non invasive tear film breakup time (NIBUT), and meibomian gland loss (MGL) investigated with Oculus Keratograph 5 M.

Results: A statistically significant relation with the soft contact lenses wearing time has been found for the indicator MGL, \% (U) $(\mathrm{p}=0.025)$. In the general study of male and female patients, a significant association was only observed in female patients $(\mathrm{p}=0.04)$. A statistically significant relation to the soft contact lenses wearing time has been found for the indicators NIBUT 1 , s ( $\mathrm{P}=0.035)$ и NIBUT Av, s ( $\mathrm{P}=0.023)$. The same relation has been found only in female patients - NIBUT $1, \mathrm{~s}$ ( $\mathrm{P}=0.035]$ и NIBUT Av, s ( $\mathrm{P}=0.023)$.

Conclusion: Long-term contact lenses wearing is proportional to the change in gland function and may contribute to the development of dry eye syndrome.
\end{abstract}

Key words: contact lens discomfort, meibomian glands, tear film break up time, dry eye syndrom

\section{INTRODUCTION}

Globally, between 140 and 150 million people wear contact lenses, with about $50 \%$ of them experiencing discomfort, especially at the end of the day (1-3). Although contact lenses are used to correct refractive abnormalities without affecting the appearance of the patient, their use causes various complications such as infections (keratitis, conjunctivitis), allergic conjunctivitis, corneal disorders and dry eye syndrome (4).

Tear film consists of three layers: mucin, aquaeous and lipid. The quality and quantity of each layer and their interactive relations are important for the tear film homeostasis (5). Meibiomian glands are lipid-producing glands

\footnotetext{
*Correspondence to: Monika Dimitrova, Tel. number: 08875625 46, e-mail: dr.m_dimitrova@abv.bg
}

located in the upper and lower eyelid. They maintain the normal stability of the tear film and are the main source of tear lipids. Changes in the lipid composition cause increased viscosity and thus consequent reduction in lipid secretion on the tear film. Meibomian dysfunction is considered to be a leading cause of the evaporative form of dry eye, which, in turn, is the most common form of dry eye syndrome (6).

Various studies have reported that people with contact lenses have a higher incidence of meibomian dysfunction (7-10). On the other hand, Ong found that the prevalence of meibomian dysfunction did not differ significantly between contact lens wearers and control subjects (11). It is still unclear how the structure and function of the meibomian glands is influenced over the years after contact lenses are put in place and what happens after their 
use ceases (12). In this respect, it is important to conduct studies aimed at the study and follow-up of changes in the meibomian glands, eyelids and tear film related to the duration of the wearing time of contact lenses.

\section{PUSPOSE}

To determine whether the wearing time of contact lenses affects the condition of the meibomian glands and the tear film breakup time.

\section{MATERIAL AND METHODS}

This is a cross-sectional study involving a total of 150 eyes in 75 patients (20 male and 55 female patients) with an age between 18 and 65 years. Five groups were allocated, with distribution criteria being the soft contact lenses wearing time: up to 5 years, from 6 to 10 years, from 11 to 20 years and more than 20 years. All subjects used soft contact lenses daily, at least 5 hours a day and 4 days a week. The patients involved in this study were studied during the period between April 2017 and June 2018.

Inclusion criteria were: over 18 years of age, visual acuity (BCVA)> 0.7 (with correction), available refractive anomaly (myopia, hypermetropia, astigmatism) to be corrected with soft contact lenses. Exclusion criteria: under 18 years of age, visual acuity $<0.7$ (with correction), corneal pathology preventing wearing of contact lenses, presence of inflammatory disease of the anterior and/or posterior segment of the eye, expected low cooperativeness on the part of the patient.
All patients underwent a thorough ophthalmic examination, including medical history, determination of visual acuity, biomicroscopy, non-invasive tear film breakup time (NIBUT), and meibomian gland loss (MGL) investigated with Oculus Keratograph $5 \mathrm{M}$ (Oculus Optikgeräte $\mathrm{GmbH}$ ), measurement of intraocular pressure, ophthalmoscopy. All subjects were asked to discontinue the use of contact lenses on the day of the visit to avoid changes in the tear film that could occur when removing the contact lenses. Ocular Keratograph $5 \mathrm{M}$ (Oculus Optikgeräte $\mathrm{GmbH}$ ) in several different modes is used to assess the condition of the ocular surface. The apparatus allows for the non-invasive measurement of tear film breakup time (NIBUT) and meibography with MGL parameter readings.

The presence of meibomian glands has been assessed using non-invasive infrared meibography. Following topical anesthetic administration, the upper and lower eyelids were everted and their infrared images were photographed using the apparatus camera. The assessment of the condition was performed objectively based on the percentage loss of meibomian glands (MGL\%) for each eyelid. The MGL\% indicator has been calculated using the following equation: $M G L(\%)=$ $($ Adrop - out / Atotal $) * 100$, where is the area of the entire eyelid and is the area in which there are no meibomian glands. (Figure 1).

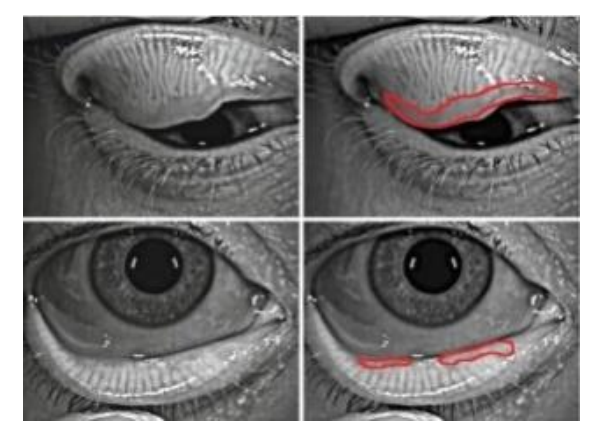

Figure 1. Image of the upper and lower eyelid meibomian glands and their percentage loss. Image 1 - outline of the entire eyelid area, and 2 - outline of the eyelid area which lacks glands.

To determine the non-invasive tear film breakup time, the patient was asked to fix a red dot with their examined eye with their head placed on the apparatus in a stationary position. Concentric circles were projected with onto the cornea using infrared mode light. The machine automatically recorded the time and place of the first tear film breakup in seconds (NIBUT 1, s). The apparatus software also calculated the mean breakup time, taking into account the values in all sectors and what percentage of the examined area remained uncovered by the tear film (NIBUT AV, s). The norm was accepted to be values over $14 \mathrm{~s}$ for NIBUT AV,s and for NIBUT 1, s-over $10 \mathrm{~s}$. 
The collected data was subjected to a Kolmogorov-Smirnov test and we found that there was an incorrect distribution, so we used the average arithmetic mean and the median in the descriptive statistics and a nonparametric method for comparative analysis of the results - a Kruskal-Wallis test. The Mann-Whitney test was also used - it is used when comparing two independent groups when there are ordinal data or when the frequency distribution pattern is different from the normal distribution pattern. For the NIBUT AV 1, s and NIBUT 1, $s$ indicators we used an Analysis of Variance (ANOVA) test to verify the equivalence of the mean values of the respective groups and the Post Hoc Tests - Tukey, which is used for pairwise comparisons after the ANOVA test. In the current work, we adopted the standard statistical confidence level $-95 \%$ confidence interval (standard error $\mathrm{p}<0.05$ ).

\section{RESULTS}

In this study, patients were distributed into five groups, with distribution criteria being the different soft contact lenses wearing time: up to 5 years (56 eyes), from 6 to 10 years (36 eyes), from 11 to 20 years ( 32 eyes) and more than 20 years ( 26 eyes). Table 1 presents the groups of patients with varying soft contact lenses wearing time and their influence on the loss of meibomian glands of upper and lower eyelids respectively. (Table 1)

Table 1. Meibomian gland loss (mean and median) in patients with different contact lenses wearing time - Kruskal-Wallis Test

\begin{tabular}{|c|c|c|c|c|c|c|c|c|c|}
\hline Gender & Indicator & $\begin{array}{c}\text { Wearing } \\
\text { time }\end{array}$ & $\mathrm{N}$ & Mean & Median & SD & Min & $\operatorname{Max}$ & $\mathrm{p}$ \\
\hline \multirow{8}{*}{ Total } & \multirow{4}{*}{$\begin{array}{c}\text { MGL, \% } \\
\text { (U-upper } \\
\text { lid) }\end{array}$} & $\leq 5$ & 56 & 13.19 & 12.50 & 13.61 & 0.00 & 58.60 & \multirow{4}{*}{0.025} \\
\hline & & $6-10$ & 36 & 19.21 & 21.25 & 18.79 & 0.00 & 76.80 & \\
\hline & & $11-20$ & 32 & 17.13 & 17.95 & 12.45 & 0.00 & 56.90 & \\
\hline & & $>20$ & 26 & 22.87 & 23.35 & 15.42 & 0.00 & 56.00 & \\
\hline & \multirow{4}{*}{$\begin{array}{l}\text { MGL, \% } \\
\text { (L-lower } \\
\quad \text { lid) }\end{array}$} & $\leq 5$ & 55 & 12.55 & 7.30 & 14.97 & 0.00 & 65.30 & \multirow{4}{*}{0.433} \\
\hline & & $6-10$ & 36 & 8.91 & 0.00 & 14.57 & 0.00 & 53.40 & \\
\hline & & $11-20$ & 32 & 12.53 & 4.25 & 17.65 & 0.00 & 65.60 & \\
\hline & & $>20$ & 26 & 12.87 & 2.45 & 15.51 & 0.00 & 41.80 & \\
\hline \multirow{8}{*}{$\begin{array}{c}\text { Male } \\
\text { patients }\end{array}$} & \multirow{4}{*}{$\begin{array}{c}\text { MGL, \% } \\
\text { (U) }\end{array}$} & $\leq 5$ & 22 & 18.46 & 20.85 & 8.82 & 0.00 & 29.50 & \multirow{4}{*}{0.844} \\
\hline & & $6-10$ & 8 & 27.76 & 20.40 & 27.18 & 0.00 & 76.80 & \\
\hline & & $11-20$ & 6 & 16.08 & 16.90 & 9.39 & 0.00 & 26.60 & \\
\hline & & $>20$ & 4 & 21.95 & 20.50 & 8.74 & 14.10 & 32.70 & \\
\hline & \multirow{4}{*}{$\begin{array}{l}\text { MGL, \% } \\
\text { (L) }\end{array}$} & $\leq 5$ & 22 & 13.87 & 9.40 & 16.25 & 0.00 & 52.60 & \multirow{4}{*}{0.791} \\
\hline & & $6-10$ & 8 & 9.08 & 4.95 & 11.10 & 0.00 & 29.60 & \\
\hline & & $11-20$ & 6 & 10.07 & 5.40 & 11.79 & 0.00 & 25.40 & \\
\hline & & $>20$ & 4 & 8.30 & 0.00 & 16.60 & 0.00 & 33.20 & \\
\hline \multirow{8}{*}{$\begin{array}{l}\text { Female } \\
\text { patients }\end{array}$} & \multirow{4}{*}{$\begin{array}{c}\text { MGL, \% } \\
\text { (U) }\end{array}$} & $\leq 5$ & 34 & 9.78 & 0.00 & 15.13 & 0.00 & 58.60 & \multirow{4}{*}{0.004} \\
\hline & & $6-10$ & 28 & 16.76 & 21.25 & 15.44 & 0.00 & 49.60 & \\
\hline & & $11-20$ & 26 & 17.37 & 17.95 & 13.21 & 0.00 & 56.90 & \\
\hline & & $>20$ & 22 & 23.04 & 23.35 & 16.49 & 0.00 & 56.00 & \\
\hline & \multirow{4}{*}{$\begin{array}{l}\text { MGL, \% } \\
\text { (L) }\end{array}$} & $\leq 5$ & 33 & 11.67 & 7.30 & 14.25 & 0.00 & 65.30 & \multirow{4}{*}{0.454} \\
\hline & & 6-10 & 28 & 8.86 & 0.00 & 15.59 & 0.00 & 53.40 & \\
\hline & & $11-20$ & 26 & 13.10 & 2.85 & 18.88 & 0.00 & 65.60 & \\
\hline & & $>20$ & 22 & 13.70 & 5.45 & 15.56 & 0.00 & 41.80 & \\
\hline
\end{tabular}

As can be seen from Table 1, a statistically significant relation with the soft contact lenses wearing time has been found for the indicator MGL, \% (U) $(p=0.025)$. In the general study of male and female patients, a significant association was only observed in female patients $(\mathrm{p}=0.04)$. The analysis revealed that in male patients there was no statistically significant relation between soft contact lenses wearing time and the indicators MGL, \% (U) and MGL\% (L). By analyzing the indicator MGL,\% (U), using the Mann-Whitney test, we compared each pair with the other ones pairwise. (Table 2) 
Table 2. Benchmarking of groups for the indicator MGL, $U \%$ - Pairwise comparisons with Mann-Whitney Test

\begin{tabular}{|c|c|c|c|c|c|c|c|}
\hline \multirow{2}{*}{ Gender } & \multirow{2}{*}{ Indicator } & $\leq 5$ & $\leq 5$ & $\leq 5$ & $6-10$ & $6-10$ & $11-20$ \\
\cline { 3 - 8 } & & $\mathrm{p}-10$ & $11-20$ & $>20$ & $11-20$ & $>20$ & $>20$ \\
\hline Total & MGL, \% (U) & 0.114 & 0.094 & $\mathbf{0 . 0 0 3}$ & 0.828 & 0.267 & 0.110 \\
\hline $\begin{array}{l}\text { Female } \\
\text { patients }\end{array}$ & MGL, \% (U) & $\mathbf{0 . 0 3 7}$ & $\mathbf{0 . 0 0 6}$ & $\mathbf{0 . 0 0 1}$ & 0.916 & 0.165 & 0.177 \\
\hline
\end{tabular}

The established relation with the wearing time of the MGL, \% (U) was determined by the significant differences between the groups with a wearing time of $\leq 5$ years and $>20$ years $(\mathrm{p}=$ 0.003). The median in the group with a wearing time of $\leq 5$ years was 12.5 and it was significantly lower than the median in the group $>20$ years (23.35). In terms of gender in females, statistically significant differences were also found between the group $\leq 5$ and all other groups - between $\leq 5$ and $6-10$ years $(\mathrm{p}=0.037), \leq 5$ and $11-20$ years $(\mathrm{p}=0.006), \leq 5$ and $>20$ years $(\mathrm{p}=0.001)$. (Table 3$)$

Table 3. NIBUT 1, $s$ and NIBUT AV, $s$ (mean and median) in patients with different contact lenses wearing time - ANOVA Test; KruskalWallis Test

\begin{tabular}{|c|c|c|c|c|c|c|c|c|c|c|c|}
\hline Gender & Indicator & $\begin{array}{c}\text { Wearing } \\
\text { time }\end{array}$ & $\mathrm{N}$ & Mean & Median & SD & Min & $\operatorname{Max}$ & $\mathrm{F}$ & $\mathrm{dfs}$ & $\mathrm{p}$ \\
\hline \multirow{8}{*}{ Total } & \multirow{4}{*}{ NIBUT 1, s I } & $\leq 5$ & 52 & 12.85 & 10.61 & 7.00 & 3.31 & 24.02 & \multirow{4}{*}{2.951} & \multirow{4}{*}{$3 ; 140$} & \multirow{4}{*}{0.035} \\
\hline & & $6-10$ & 34 & 8.87 & 7.22 & 5.82 & 2.61 & 23.90 & & & \\
\hline & & $11-20$ & 32 & 9.96 & 7.97 & 6.54 & 2.17 & 24.02 & & & \\
\hline & & $>20$ & 26 & 10.16 & 9.50 & 6.48 & 2.02 & 24.02 & & & \\
\hline & \multirow{4}{*}{ NIBUT Av, s I } & $\leq 5$ & 52 & 16.38 & 17.10 & 6.35 & 3.31 & 24.02 & \multirow{4}{*}{3.282} & \multirow{4}{*}{$3 ; 140$} & \multirow{4}{*}{0.023} \\
\hline & & $6-10$ & 34 & 12.47 & 10.49 & 5.75 & 4.73 & 23.90 & & & \\
\hline & & $11-20$ & 32 & 13.80 & 13.51 & 5.94 & 2.53 & 24.02 & & & \\
\hline & & $>20$ & 26 & 15.39 & 17.11 & 5.60 & 4.26 & 24.10 & & & \\
\hline \multirow{8}{*}{$\begin{array}{c}\text { Male } \\
\text { patients }\end{array}$} & \multirow{4}{*}{ NIBUT 1, s I } & $\leq 5$ & 18 & 10.36 & 8.67 & 6.60 & 3.31 & 24.02 & & & \multirow{4}{*}{$0.597 *$} \\
\hline & & 6-10 & 8 & 8.61 & 4.59 & 6.70 & 3.57 & 21.35 & & & \\
\hline & & $11-20$ & 6 & 13.69 & 13.38 & 7.77 & 2.74 & 23.77 & & & \\
\hline & & $>20$ & 4 & 12.83 & 14.15 & 6.95 & 3.76 & 19.25 & & & \\
\hline & \multirow{4}{*}{ NIBUT Av, s I } & $\leq 5$ & 18 & 15.82 & 17.29 & 7.23 & 3.31 & 24.02 & & & \multirow{4}{*}{$0.586^{*}$} \\
\hline & & 6-10 & 8 & 13.00 & 9.98 & 6.79 & 4.73 & 23.20 & & & \\
\hline & & $11-20$ & 6 & 17.02 & 15.23 & 4.88 & 12.46 & 23.77 & & & \\
\hline & & $>20$ & 4 & 16.84 & 16.91 & 4.37 & 11.42 & 22.11 & & & \\
\hline \multirow{5}{*}{$\begin{array}{l}\text { Female } \\
\text { patients }\end{array}$} & \multirow{4}{*}{ NIBUT 1, s I } & $\leq 5$ & 34 & 14.17 & 14.72 & 6.94 & 3.89 & 24.02 & \multirow{4}{*}{4.792} & \multirow{4}{*}{$3 ; 104$} & \multirow{4}{*}{0.004} \\
\hline & & 6-10 & 26 & 8.95 & 7.35 & 5.67 & 2.61 & 23.90 & & & \\
\hline & & $11-20$ & 26 & 9.11 & 7.01 & 6.07 & 2.17 & 24.02 & & & \\
\hline & & $>20$ & 22 & 9.67 & 9.21 & 6.45 & 2.02 & 24.02 & & & \\
\hline & NIBUT Av, s I & $\leq 5$ & 34 & 16.67 & 17.03 & 5.93 & 6.25 & 24.02 & 3.406 & $3 ; 104$ & 0.020 \\
\hline
\end{tabular}

As can be seen from Table 3, a statistically significant relation to the soft contact lenses wearing time has been found for the indicators NIBUT $1, \mathrm{~s}[\mathrm{~F}(3 ; 14)=2.95, \mathrm{P}=0.035]$ и NIBUT Av, $s[F(3 ; 14)=3.28, P=0.023]$. As established in the ANOVA analysis, a statistically significant relation between the soft contact lenses wearing time and the indicators NIBUT $1, \mathrm{~s}$
$[\mathrm{F}(3 ; 10)=2.95, \quad \mathrm{P}=0.035] \quad$ и $\quad$ NIBUT $\mathrm{Av}, \mathrm{s}$ $[F(3 ; 10)=3.28, P=0.023]$ has been found only in female patients. For this reason, pairwise comparisons of the corresponding groups according to the wearing time of contact lenses and the two indicators have been carried out only for females. (Table 4) 
DIMITROVA M., et al.

Table 4. Benchmarking of groups for the indicators NIBUT 1, s and NIBUT Av, s-Pairwise comparisonsTukey HSD test

\begin{tabular}{|c|c|c|c|c|c|c|c|}
\hline \multirow{2}{*}{ Gender } & Indicator & $\leq 5$ & $\leq 5$ & $\leq 5$ & $6-10$ & $6-10$ & $11-20$ \\
\cline { 3 - 7 } & & $6-10$ & $11-20$ & $>20$ & $11-20$ & $>20$ & $>20$ \\
\cline { 2 - 7 } & NIBUT 1, s I & $\mathbf{0 . 0 3 3}$ & 0.206 & 0.319 & 0.905 & 0.875 & 0.997 \\
\hline Total & NIBUT Av, s I & $\mathbf{0 . 0 1 9}$ & 0.226 & 0.903 & 0.805 & 0.245 & 0.745 \\
\hline Total & NIBUT 1, s I & $\mathbf{0 . 0 1 1}$ & $\mathbf{0 . 0 1 4}$ & 0.052 & $\mathbf{0 . 0 1 1}$ & 0.980 & 0.960 \\
\hline $\begin{array}{c}\text { Female } \\
\text { patients }\end{array}$ & NIBUT Av, s I & $\mathbf{0 . 0 2 5}$ & 0.088 & 0.769 & $\mathbf{0 . 0 2 5}$ & 0.967 & 0.611 \\
\hline $\begin{array}{c}\text { Female } \\
\text { patients }\end{array}$ & & & & & & $\mathrm{p}$ \\
\hline
\end{tabular}

As can be seen from Table 4, the pairwise comparison revealed a statistically significant difference between the values of the NIBUT 1 , $\mathrm{s}$ and NIBUT Av, s parameters studied, upon benchmarking of the groups with a soft contact lenses wearing time $<5$ years and the one with a wearing time between 6 and 10 years. When comparing the groups with soft contact lenses wearing time $<5$ years with that of between 11 and 20 years, only for the indicator the NIBUT 1 , s a statistically significant difference has been found $(p=0.014)$. Comparing groups of 6 to 10 years and 11 to 20 years, a statistically significant difference has found in the indicators NIBUT $1, \mathrm{~s}(\mathrm{p}=0.011)$ and NIBUT Av, s $(\mathrm{p}=0.025)$.

\section{DISCUSSION}

In the present study, in-depth examinations of the meibomian glands were performed in people with varying contact lenses wearing time using a new contactless technique. The results showed that persons wearing contact lenses for a longer period of time had significantly greater loss of meibomian glands compared to patients with shorter wearing time. This suggests that the meibomian gland loss is one of the mechanisms underlying CLrelated dry eye. The condition of the meibomian glands in patients with contact lenses was examined in many studies. In the study of Arita et al., the data did not show that the extent of dropping of the meibomian glands was related to the dry eye condition in patients wearing contact lenses (4). This study, however, examines the meibomian glands only in the central part of the lower eyelid, until our explores the meibomian glands on the upper and lower eyelid. These results indicate that changes in long-term wearing of contact lenses were observed only in the meibomian glands of the upper eyelid. Thus, the absence of these glands only in the central lower eyelid does not necessarily represent the full extent of changes in the meibomian glands, indicating that these results are likely to be more reliable than the ones previously published (4). Ong and Larke proved that the melting point of meibome in people wearing contact lenses for many years was higher by $3^{\circ} \mathrm{C}$ than that of patients with shorter wearing time (13). Unlike these observations, Pucker et al. found that the production and quality of meibome did not change in people with contact lenses (14).

Two hypotheses have been proposed as a cause of the loss of meibomian glands due to the long-term use of contact lenses (4). Ong and Larke suggest that mechanical contact lens trauma causes clogging of the meibomian gland canals (13). However, Henriquez and Korb suggested that meibomian gland dysfunction is a result of the aggregation of desquamated epithelial cells at the orifices of the glands (15). In most of the CL wearers with meibomian gland changes, the clusters of meibomian glands were similarly shortened (4). Moreover, the shortening of the meibomian glands in the contact lens wearers started from the distal side. These results suggest that the chronic irritation of meibomian glands from contact lenses caused by the conjunctive was a causal mechanism for changes in their conditions in people wearing contact lenses (4). This hypothesis is also consistent with our study that the loss of meibomian glands depends on the contact lenses wearing time. We also found similar results when examining the breakup time of the tear film. According to Arita et al., this indicator significantly differs in people with long-term contact lenses wearing time compared to patients with a shorter time (5). Sengor et al. reported results similar to ours, 
finding that in the longer contact lenses wearing time the tear film breakup time was shorter (16). An interesting study by Algambi et al. showed that changes in the meibomian glands and the shortened tear film breakup occurred in the second year of wearing contact lenses (12), which is somewhat different from our data. Some authors consider that because of the more variable features of the tear film, its assessment cannot always be sufficiently precise (16).

\section{CONCLUSION}

Many studies have examined whether longterm use of contact lenses influences the condition of the meibomian glands. Our work has shown that their long-term wearing is proportional to the change in gland function and may contribute to the development of dry eye syndrome in patients wearing contact lenses. Dry eye syndrome, due to the increased evaporation of the tear film, is more prevalent in the longer-term use of contact lenses.

\section{REFERENCES}

1. Cavanagh,H.D. et al., Castroviejo Lecture 2009: 40 years in search of the perfect contact lens, Cornea, 29 (10):1075-1085, 2010.

2. Stapleton, F., et al., The epidemiology of contact lens related infiltrates, Optom Vis Sci, 84(4):257-272, 2007.

3. Richdale, K., et al., Frequency of and factors associated with contact lens dissatisfaction and discontinuation, Cornea 26 (2):168-174, 2007.

4. Arita, R., Itoh, K., Inoue, K., Kuchiba, A., Yamaguchi, T., \& Amano, S. Contact Lens Wear Is Associated with Decrease of Meibomian Glands. Ophthalmology, 116(3):379-384, 2009.

5. Arita, R., Fukuoka, S., \& Morishige, N., Meibomian Gland Dysfunction and Contact Lens Discomfort. Eye \& Contact Lens: Science \& Clinical Practice, 43(1):17-22, 2017.

6. Green-Church, KB, Butovich I, Willcox M et al., The international workshop on meibomian gland dysfunction: report of the subcommittee on tear film lipids and lipidprotein interactions in health and disease. Invest Ophthalmol Vis Sci, 52:1979-1993, 2011.

7. Villani, E., et al., In vivo confocal microscopy of meibomian glands in contact lens wearers, Invest Ophthalmol Vis Sci, 52 (8):5215-5219, 2011.

8. Ong, B.L., Larke J.R., Meibomian gland dysfunction: some clinical, biochemical and physical observations, Ophthalmic Physiol Opt., 10(2):144-148, 1990.

9. Henriquez ,A.S., Korb D.R., Meibomian glands and contact lens wear, $\mathrm{Br} J$ Ophthalmol, 65(2):108-111, 1981.

10.Korb, D.R., Henriquez A.S., Meibomian gland dysfunction and contact lens intolerance, J Am Optom Assoc, 51(3):243251, 1980.

11.Ong, BL. Relation between contact lens wear and meibomian gland dysfunction. Optom Vis Sci, 73:208-10, 1996.

12.Alghamdi, W. M., Markoulli, M., Holden, B. A., \& Papas, E. B., Impact of duration of contact lens wear on the structure and function of the meibomian glands. Ophthalmic and Physiological Optics, 36(2):120-131, 2016.

13.Ong BL, Larke JR., Meibomian gland dysfunction: Some clinical, biochemical and physical observations. Ophthalmic Physiol Opt., 10:144-148, 1990.

14.Pucker, A. D., Jones-Jordan, L. A., Li, W., Kwan, J. T., Lin, M. C., Sickenberger, W., Jones, L. W., Associations with Meibomian Gland Atrophy in Daily Contact Lens Wearers. Optometry and Vision Science, 92(9):206-213, 2015.

15.Henriquez AS, Korb DR., Meibomian glands and contact lens wear. $\mathrm{Br} J$ Ophthalmol, 65:108-11, 1981.

16.Sengor, T., Kurna, S. A., Ozbay, N., Ertek, S., Aki, S., \& Altun, A., Contact LensRelated Dry Eye and Ocular Surface Changes with Mapping Technique in LongTerm Soft Silicone Hydrogel Contact Lens Wearers. European Journal of Ophthalmology, 22(7):17-23, 2012. 